Z. Epileptol. $2021 \cdot 34: 42-48$

https://doi.org/10.1007/s10309-020-00390-0

Angenommen: 10. Dezember 2020

Online publiziert: 29. Dezember 2020

(c) Der/die Autor(en) 2021

\section{Die in der Regel weniger gut loka- lisierende Semiologie, die diffusen EEG-Befunde sowie die Schwie- rigkeiten in der MR-Abgrenzung epileptogener Läsionen in den ersten Lebensjahren machen die Epilepsiechirurgie bei Kindern zu einer Herausforderung. Dies trifft besonders auf epileptogene Läsio- nen im posterioren Kortex zu, welche aber dennoch mit angemessener Abklärung und Patientenselekti- on erfolgreich epilepsiechirurgisch behandelt werden können.}

\section{Epilepsiechirurgie: Eine wirksame Therapie}

Die Epilepsie ist mit einer Inzidenz von $1 \%$ eine der häufigsten neurologischen Erkrankungen im Kindes- und Jugendalter, mit einem Inzidenzmaximum im ersten Lebensjahr [1]. Kohortenstudien haben gezeigt, dass trotz antikonvulsiver Behandlung 20-25\% aller Kinder mit Epilepsie weiterhin persistierende epileptische Anfälle zeigen, und 10-15\% formal die Kriterien einer Pharmakoresistenz erfüllen [2]. Bei fehlenden Hinweisen auf ein Epilepsiesyndrom weist eine früh im Verlauf eintretende Pharmakoresistenz mit hoher Wahrscheinlichkeit auf die Anwesenheit einer strukturellen Hirnläsion hin [3]. Eine Pharmakoresistenz geht oft mit relevanten Komorbiditäten einher, welche kognitive Entwicklungsdefizite, Verhaltensstörungen, psychiatrische Störungen (einschließlich Depression und Angststörung), starke Einschränkungen in der Lebensqualität sowie ein erhöhtes $\operatorname{SUDEP}($ „sudden unexpected death in epilepsy“)-Risiko umfassen [4].

Richard E. Rosch - Andrea Rüegger · Georgia Ramantani

Neuropädiatrie, Universitäts-Kinderspital Zürich, Zürich, Schweiz

\title{
Pädiatrische Epilepsiechirurgie bei posterioren Epilepsien
}

Historisch wurde die Epilepsiechirurgie als eine Behandlungsmöglichkeit der letzten Instanz angesehen. Jedoch ist die Anzahl epilepsiechirurgischer Eingriffe bei Kindern im Gegensatz zu Erwachsenen im letzten Jahrzehnt deutlich angestiegen [5-8]. Ein epilepsiechirurgischer Eingriff ist bei entsprechend ausgewählten Patienten der Behandlungsstandard und ist der Behandlung mit einem dritten Medikament auch in randomisierten kontrollierten Studien überlegen [9]. Es hat sich außerdem gezeigt, dass ein epilepsiechirurgischer Eingriff die negativen Auswirkungen von persistierenden epileptischen Anfällen und jahrelanger Pharmakotherapie abwenden kann [10, 11]. Dadurch wird die kognitive Entwicklung der betroffenen Kinder begünstigt $[12,13]$, oder zumindest werden weitere kognitive Einbußen verhindert [14-18]. Auch Morbidität und Mortalität werden durch die Epilepsiechirurgie gesenkt. So konnte gezeigt werden, dass Verzögerungen in der Durchführung eines epilepsiechirurgischen Eingriffes das Risiko einer ausbleibenden Anfallskontrolle, einer kognitiven Entwicklungsverzögerung und sogar das Todesrisiko erhöhen $[19,20]$.

\section{Prächirurgische Abklärung bei pädiatrischen Patienten}

Das Hauptziel der Epilepsiechirurgie ist die Resektion oder Diskonnektion der epileptogenen Zone, wobei eloquente Hirnareale und deren Funktionen geschont werden sollen. Die epileptogene Zone wird definiert als das kortikale Areal, das für die Anfallsentstehung zuständig ist und dessen Entfernung notwendig und ausreichend ist, um Anfallsfreiheit zu erreichen [21]. Die epileptogene Zo- ne kann der Ausdehnung einer MRabgrenzbaren Hirnläsion entsprechen, sie kann sich jedoch ggf. über die Läsion hinaus erstrecken [22]. Ein epilepsiechirurgischer Eingriff ist grundsätzlich indiziert, wenn die Anfallssemiologie, die EEG-Befunde und die Bildgebung eine eindeutige Definition des epileptogenen Areals erlauben und eine Resektion dieses Areals ohne inakzeptable neurologische und neuropsychologische Defizite möglich ist.

Trotz der großen Fortschritte in der pädiatrischen prächirurgischen Epilepsiediagnostik stellt die Identifikation der epileptogenen Zone sowie der eloquenten Kortexareale oft - insbesondere in den ersten Lebensjahren - eine große Herausforderung dar. Die EEG-Auffälligkeiten bei jungen Kindern sind häufig auch bei fokalen Epilepsien ausgedehnt, multifokal oder bilateral und somit weniger aufschlussreich bezüglich der Lokalisation des Anfallsursprungs als bei Jugendlichen oder Erwachsenen [14, 23-25]. Darüber hinaus ist die Anfallssemiologie bei fokalen Anfällen in den ersten Lebensjahren kaum lokalisierend, auch weil die Bewusstseinslage häufig nur schwer beurteilbar ist [26]. Die Interpretation der MR-Bildgebung ist aufgrund der unreifen Myelinisierung des Marklagers in den ersten 2 Lebensjahren mit großen Schwierigkeiten verbunden [27], sodass wiederholte MRUntersuchungen notwendig sind. Auch die Identifikation von eloquenten Hirnarealen stellt eine Herausforderung dar, zumal entsprechende Untersuchungen (fMRI, Wada-Test) eine möglichst gute Kooperation des Kindes voraussetzen.

Dennoch haben technologische Fortschritte sowie der Erfahrungsgewinn der letzten Jahrzehnte das Indikationsspek- 

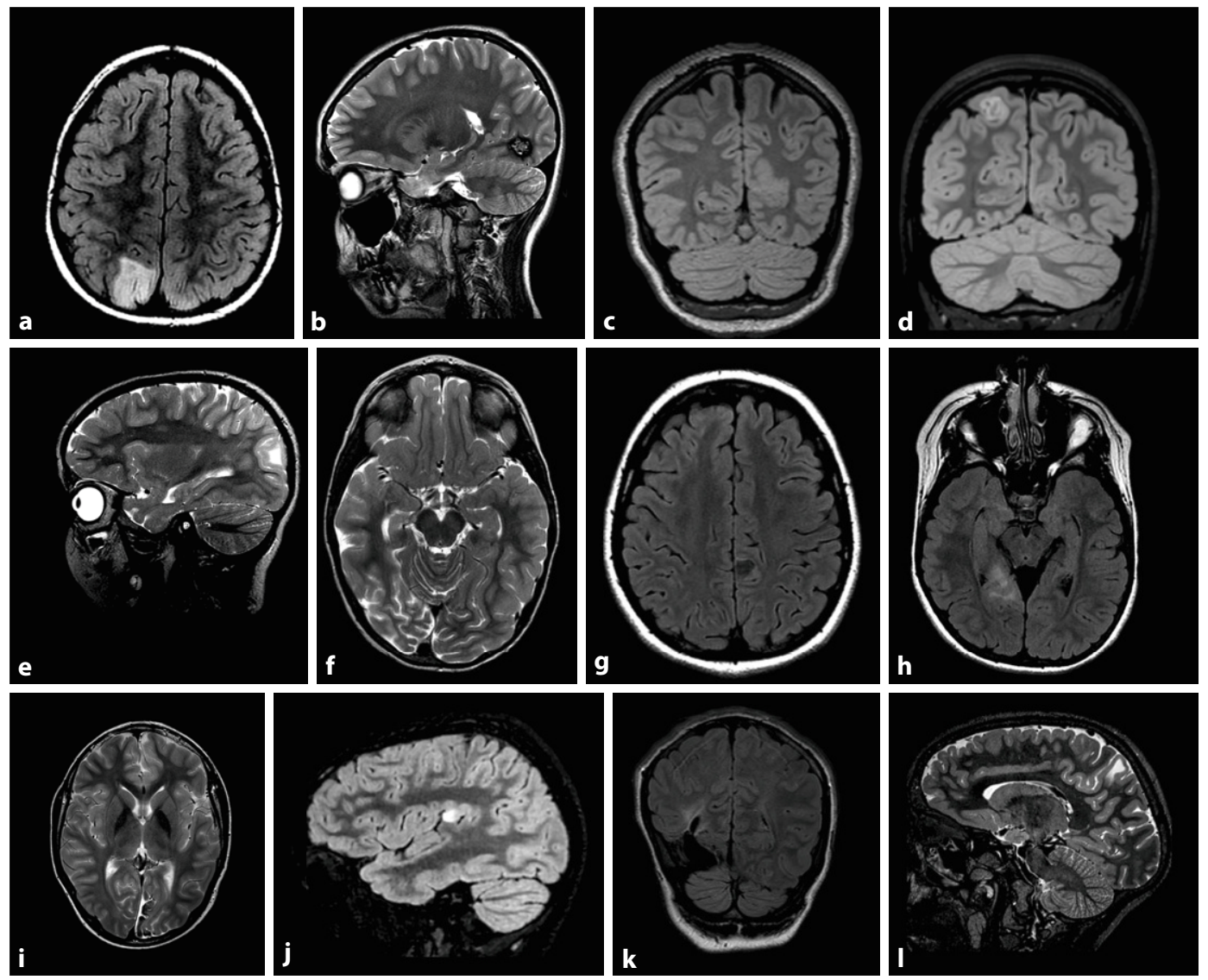

Abb. $1<$ a Diffuses Astrozytom rechts okzipital. b Kavernom okzipital. c FCD 2a links okzipital. d Gangliogliom rechts parietal. e FCD 2b okzipital. fSturge-Weber-Syndrom rechts temporookzipital. g DNET links parietal mesial. h FCD temporookzipital mesial. i Sturge-WeberSyndrom links parietookzipital.j FCD im Gyrus supramarginalis. $\mathbf{k}$ Perinatale Hirnblutung rechts temporookzipital.I DNET parietal

trum der pädiatrischen Epilepsiechirurgie erweitert und die Vorgehensweise drastisch verändert. Auch Kinder und Jugendliche mit ausgedehnten, diffusen EEG-Auffälligkeiten und ausgedehnten multifokalen oder multilobären Läsionen können heute nach einem epilepsiechirurgischen Eingriff anfallsfrei werden [14, 24, 25, 28, 29].

\section{Posteriore Resektionen - bei Kindern besonders relevant}

Während bei Erwachsenenkohorten die Temporallappenepilepsie überwiegt, werden extratemporale und temporale Epilepsien bei Kindern in etwa gleich häufig gesehen [30]. Resektionen des posterioren Kortex werden in etwa $10 \%$ der Fälle durchgeführt [30]. Diese posterioren Resektionen können $\mathrm{Pa}$ rietallappen, Okzipitallappen und zum Teil die posterioren Randgebiete des Temporallappens beinhalten. Extratemporale Resektionen sind insgesamt nicht so erfolgreich wie temporale Resektionen [28], da sowohl die Lokalisierung der epileptogenen Zone als auch der Erhalt von funktionell relevanten Arealen (eloquenter Kortex) besondere Herausforderungen darstellen, besonders auch bei jüngeren Kindern. Mehrere Studien unterstreichen die erschwerte Abgrenzbarkeit der epileptogenen Zone besonders auch innerhalb der parietookzipitalen und hinteren temporalen Regionen bei oft unspezifischer Anfallssemiologie, die aufgrund der raschen Propagation fälschlicherweise in die vorderen Hirnregionen lokalisiert werden kann, was auch von Jacobs et al. in dieser Ausgabe näher besprochen wird. Im Kindesalter wird diese Herausforderung noch größer, da die elektroenzephalographischen Auffälligkeiten oftmals diffus oder generalisiert und somit zur Eingrenzung der epileptogenen Zone nicht wegweisend sind [19].

Trotz dieser Herausforderungen bei der operativen Behandlung extratemporaler Epilepsien hat sich die Epilepsiechirurgie als eine wichtige therapeutische Option für ausgewählte Patienten erwiesen. Laut einer Metaanalyse zur pädiatri- schen extratemporalen Epilepsiechirurgie [31] wurden $56 \%$ der Kinder und Jugendlichen unabhängig von der Lokalisation der epileptogenen Zone nach dem Eingriff anfallsfrei (Engel I). Außerdem zeigt sich, dass die kürzere Latenz zwischen Epilepsiebeginn und Operation sowie die vollständige Resektion MR-nachweisbarer epileptogener Läsionen die Chancen auf eine postoperative Anfallsfreiheit signifikant erhöhen, insbesondere bei Patienten mit tumorassoziierten Epilepsien.

Die Ätiologie posteriorer struktureller Epilepsien ist vielseitig (s. auch Kasper et al. in diesem Heft), gerade auch bei Kindern (-Abb. 1). Fokale kortikale Dysplasien („focal cortical dysplasias“ [FCD]) sind die häufigste Ätiologie und benötigen oft eine ausgiebige prächirurgische Abklärung, einschließlich invasiver EEG-Ableitung, um die Resektionsgrenze korrekt zu setzen und postoperative Anfallsfreiheit zu gewährleisten [15, 19, 32, 33]. Epilepsien, die von FCDs innerhalb des frontalen, parietalen oder okzipitalen Neokortex hervorgerufen wer- 
den, neigen zudem zu einer Reorganisation des Netzwerkes. Diese kann dazu führen, dass Anfälle postoperativ nach einiger Zeit wieder auftreten können [34].

\section{Postoperativer Verlauf nach posterioren Resektionen}

Seit den 1990er-Jahren hat sich der Anteil an Patienten, die nach einem epilepsiechirurgischen Eingriff im posterioren Kortex anfallsfrei werden, von 43-47\% auf $61-75 \%$ im letzten Jahrzehnt gesteigert $[35,36]$. Diese drastische Verbesserung des postoperativen Verlaufes für viele Patienten hängt v. a. mit den neuesten Entwicklungen in der Bildgebung zusammen, die dazu geführt haben, dass der Anteil an MR-negativen Patienten von $13 \%$ auf nur $5 \%$ innerhalb des letzten Jahrzehnts abgesunken ist. Die Literatur zu Epilepsie des posterioren Kortex ist vergleichsweise spärlich und teilweise widersprüchlich, was der relativ niedrigen Inzidenz von posterioren Läsionen im Vergleich zu anderen kortikalen Arealen entspricht. Dennoch gibt es mittlerweile mehrere Kohortenstudien inklusive einzelner kürzlich publizierter pädiatrischer Studien [19, 37], welche ein neues Licht auf dieses weniger ausführlich beschriebene Gebiet werfen und in • Tab. 1 zusammengefasst sind.

Insgesamt wurden in den Studien in - Tab. 1 mehr als 450 Kinder mit einer posterioren kortikalen Epilepsie postoperativ nachverfolgt. Von diesen waren knapp 300 (65\%) bis zu einer mindestens 1-jährigen Verlaufskontrolle anfallsfrei. Für die meisten Kohorten war FCD der häufigste histopathologische Befund mit Tumoren oft an zweiter Stelle. Statistisch mit Anfallsfreiheit assoziierte Faktoren waren divers und zum Teil zwischen Studien auch widersprüchlich. Gehäuft traten jedoch Faktoren auf, die eine eindeutige Lokalisierung suggerieren (z.B. lateralisierende Semiologie, Abwesenheit kontralateraler oder anders lokalisierter Befunde), eine vollständige Resektion indizieren (keine residuelle Abnormität im MRT, keine epilepsietypischen Potenziale im postoperativen EEG) oder mit einem weniger ausgedehnten epileptogenen Netzwerk assoziiert sind (z.B.

Z. Epileptol. 2021 · 34:42-48 https://doi.org/10.1007/s10309-020-00390-0

(c) Der/die Autor(en) 2021

\section{R. E. Rosch · A. Rüegger · G. Ramantani}

\section{Pädiatrische Epilepsiechirurgie bei posterioren Epilepsien}

\section{Zusammenfassung}

Hintergrund. Die Epilepsiechirurgie hat sich als wirksame Behandlungsmethode für pharmakoresistente, strukturelle Epilepsien erwiesen. Läsionen im posterioren Kortex stellen dabei - gerade bei Kindern - eine besondere Herausforderung dar.

Fragestellung. Wirksamkeit der epilepsiechirurgischen Eingriffe bei Kindern und Jugendlichen mit posterioren strukturellen Läsionen.

Material und Methoden. Literaturüberblick und Zusammenfassung der hauptsächlichen Befunde publizierter Studien zu pädiatrischen Kohorten nach epilepsiechirurgischer Behandlung posteriorer Epilepsien. Ergebnisse. Epilepsien aufgrund posteriorer Läsionen sind bei Kindern häufiger als bei Erwachsenen und sind oft weniger klar lokalisiert und weniger deutlich von eloquentem Kortex abgrenzbar. Dennoch haben sich die postoperativen Verläufe in den letzten Jahrzehnten auf Grund von Fortschritten in der Abklärung deutlich verbessert.

Schlussfolgerung. Auch bei posterioren epileptogenen Läsionen ist die Epilepsiechirurgie oft eine wirksame Behandlungsmethode. Dabei bestehen die besten Aussichten auf Anfallsfreiheit nach frühzeitigen, ausreichend ausgedehnten Eingriffen. Das heißt, diese Kinder und Jugendlichen müssen für ein optimales Outcome umfangreich und v.a. frühzeitig abgeklärt werden.

\section{Schlüsselwörter}

Postoperativer Verlauf - Extratemporale Epilepsie · Fokale kortikale Dysplasien . Pädiatrie · Prächirurgische Abklärung

\section{Pediatric epilepsy surgery for posterior epilepsies}

\section{Abstract}

Background. Epilepsy surgery has proven to be an effective treatment strategy for pharmacoresistant structural epilepsy. Lesions in the posterior cortex are particularly challenging in their evaluation for surgery, especially in children.

Objective. Study of the effectiveness of surgical treatment for children and adolescents with posterior structural lesions. Material and methods. Literature review and summary of the key findings of published studies on pediatric cohorts following surgical treatment for posterior cortex epilepsy. Results. Epilepsy caused by lesions in the posterior cortex is more common in children than in adults and is often less clearly associated with localizing symptoms and difficult to delineate from eloquent cortex. Despite these issues, outcomes have significantly improved over the last decades thanks to advances in presurgical evaluation. Conclusion. Epilepsy surgery is an effective treatment for epileptogenic lesions in the posterior cortex. The best prospects for freedom from seizures are after early and sufficiently extensive interventions, mandating an early and thorough evaluation in affected children and adolescents.

\section{Keywords}

Postsurgical outcome $\cdot$ Extratemporal epilepsy - Focal cortical dysplasia - Pediatrics. Presurgical evaluation intralobäre Läsionen, Epilepsiebeginn nach dem frühen Kindesalter).

Eine kürzlich publizierte größere, rein pädiatrische deutsche Serie schloss 50 Kinder und Jugendliche mit ein [19], von denen alle bis auf einen MRIpositiv waren und bei $26 \%$ eine invasive Abklärung durchgeführt wurde. Postoperativ blieben $60 \%$ der Patienten anfallsfrei bis zum letzten Kontrolltermin, der durchschnittlich 8 Jahre nach dem Eingriff stattfand. Bei einem Drittel konnten die Antikonvulsiva abgesetzt werden. Auch in dieser Kohorte waren FCDs die häufigste histopathologische Diagnose (42\%), gefolgt von Tumoren $(32 \%)$. In dieser Studie stellten sich linksseitige und parietal gelegene epileptogene Zonen und Resektionen als unabhängige Risikofaktoren für ein Anfallsrezidiv heraus. Eine längere Dauer der Epilepsie bis zum Eingriff wurde als einziger unabhängiger Risikofaktor für ein Anfallsrezidiv identifiziert. 
Tab. 1 Epilepsiechirurgische pädiatrische Kohortenstudien zu Patienten mit posteriorer Epilepsie

\begin{tabular}{|c|c|c|c|c|}
\hline Artikel & Patienten & $\begin{array}{l}\text { Resektionen und Histo- } \\
\text { pathologie }\end{array}$ & $\begin{array}{l}\text { Invasive } \\
\text { Ablei- } \\
\text { tungen }\end{array}$ & Mit Anfallsfreiheit assoziierte Faktoren \\
\hline $\begin{array}{l}\text { Boesebeck } \\
{[51](2002)} \\
\text { Brain } \\
\text { Deutschland }\end{array}$ & $\begin{array}{l}41 \mathrm{Pt}^{\mathrm{a}} \text {, gemischt } \\
\text { Alter: } 13-53(29) \\
\text { Rekr: } 1991-1999 \\
\text { Nachkontr: } 2 \mathrm{~J}\end{array}$ & $\begin{array}{l}\text { P, O, postT } \\
24 \% \mathrm{FCD}, 24 \% \text { Tumor, } \\
41 \% \text { Gliose } \\
{[0 \% \mathrm{MRI} \text { negativ] }}\end{array}$ & $\begin{array}{l}57 \% \text { chr. } \\
\text { iEEG } \\
\text { n/a akut } \\
\text { ECoG }\end{array}$ & $\begin{array}{l}\text { Anfallsfrei } \mathbf{4 5} \% \text {, Med. gestoppt: } \mathrm{n} / \mathrm{a} \\
\text { Semiologie: lateralisierende Auren, lateralisierende Anfälle } \\
\text { EEG: keine ETP postoperativ } \\
\text { Ätiologie: Tumor }\end{array}$ \\
\hline $\begin{array}{l}\text { Barba [52] } \\
(2005) \\
\text { J Neurol } \\
\text { Italien }\end{array}$ & $\begin{array}{l}14 \text { Pt, gemischt } \\
\text { Alter: } 8-29(19) \\
\text { Rekr: } 1997-2000 \\
\text { Nachkontr: } \mathrm{n} / \mathrm{a}\end{array}$ & $\begin{array}{l}\mathrm{P}, \mathrm{O} \text {, postT } \\
14 \% \mathrm{FCD}, 29 \% \text { Tumor, } \\
24 \% \text { vaskulär, } 14 \% \text { Gliose } \\
{[7 \% \mathrm{MRI} \text { negativ] }}\end{array}$ & $\begin{array}{l}36 \% \text { chr. } \\
\text { iEEG } \\
79 \% \text { akut } \\
\text { ECoG }\end{array}$ & $\begin{array}{l}\text { Anfallsfrei } 45 \% \text {, Med. gestoppt: } \mathrm{n} / \mathrm{a} \\
\text { EEG: fokale Anfälle (Trend: fokale interiktale Aktivität im iEEG) } \\
\text { Operation: komplette Resektion }\end{array}$ \\
\hline $\begin{array}{l}\text { Dalmagro } \\
\text { [53] (2005) } \\
\text { Epilepsia } \\
\text { Brasilien }\end{array}$ & $\begin{array}{l}43 \text { Pt, gemischt } \\
\text { Alter: } \mathrm{n} / \mathrm{a}(20) \\
\text { Rekr: } 1994-2003 \\
\text { Nachkontr: } 3,3 \mathrm{~J}\end{array}$ & $\begin{array}{l}\text { P, O, postT } \\
45 \% \text { FCD, } 14 \% \text { Tumor, } \\
20 \% \text { Gliose } \\
{[0 \% \text { MRI negativ] }}\end{array}$ & $\begin{array}{l}76 \% \text { chr. } \\
\text { iEEG } \\
\text { n/a akut } \\
\text { ECoG }\end{array}$ & $\begin{array}{l}\text { Anfallsfrei } 71 \text { \%, Med. gestoppt: } \mathrm{n} / \mathrm{a} \\
\text { Anamnese: neurologisch unauffällig, kürzere Dauer der Epilepsie, gerin- } \\
\text { gere Anfallsfrequenz } \\
\text { Ätiologie: keine Gliose bei der Histologie }\end{array}$ \\
\hline $\begin{array}{l}\text { Jehi [54] } \\
\text { (2009) } \\
\text { Epilepsia } \\
\text { USA }\end{array}$ & $\begin{array}{l}57 \text { Pt, gemischt } \\
\text { Alter: 0,3-55 }(20,4) \\
\text { Rekr: 1994-2006 } \\
\text { Nachkontr: 3,3 J }\end{array}$ & $\begin{array}{l}\mathrm{P}, \mathrm{O}, \mathrm{PO} \\
34 \% \mathrm{FCD}, 39 \% \text { Tumor, } \\
25 \% \text { vaskulär } \\
{[6 \% \mathrm{MRI} \text { negativ] }}\end{array}$ & $\begin{array}{l}42 \% \text { chr. } \\
\text { iEEG } \\
33 \% \text { akut } \\
\text { ECoG }\end{array}$ & $\begin{array}{l}\text { Anfallsfreiheit } 67 \% \text {, Med. gestoppt: } 23 \% \\
\text { Anamnese: jüngeres Alter bei Operation } \\
\text { EEG: keine präoperativen ipsilateralen temporalen Sharp-Waves, rasche } \\
\text { Aktivität bei Anfallsbeginn (iEEG), keine postoperativen ipsilateralen } \\
\text { Sharp-Waves } \\
\text { MRI: sichtbare Läsion } \\
\text { Operation: parietale Resektionen, komplette Resektion, ausgedehntere } \\
\text { Resektion }\end{array}$ \\
\hline $\begin{array}{l}\text { Yu [55] (2008) } \\
\text { Seizure } \\
\text { China }\end{array}$ & $\begin{array}{l}43 \text { Pt, gemischt } \\
\text { Alter: } 4-43(20,3) \\
\text { Rekr: } 2001-2006 \\
\text { Nachkontr: } 2,8 \mathrm{~J}\end{array}$ & $\begin{array}{l}\mathrm{P}, \mathrm{O}, \mathrm{PO}, \text { post T } \\
42 \% \mathrm{FCD}, 7 \% \text { Tumor, } \\
29 \% \text { vaskulär, } 5 \% \text { Gliose }\end{array}$ & $\begin{array}{l}51 \% \text { chr. } \\
\text { iEEG } \\
100 \% \text { akut } \\
\text { ECoG }\end{array}$ & $\begin{array}{l}\text { Anfallsfreiheit } 61 \% \text {, Med. gestoppt } \mathrm{n} / \mathrm{a} \\
\text { keine eindeutigen prädiktiven Faktoren }\end{array}$ \\
\hline $\begin{array}{l}\text { Ibrahim [35] } \\
\text { (2012) } \\
\text { Epilepsy Res } \\
\text { Kanada }\end{array}$ & $\begin{array}{l}41 \text { Pt, pädiatrisch } \\
\text { Alter: } 1-18(10,5) \\
\text { Rekr: } 2000-2010 \\
\text { Nachkontr: } 3,1 \mathrm{~J}\end{array}$ & $\begin{array}{l}\text { O, PO, TO, TPO } \\
29 \% \text { FCD, } 16 \% \text { Tumor, } \\
17 \% \text { Gliose, } 2 \% \text { tuberöse } \\
\text { Sklerose, } 10 \% \text { vaskulär } \\
\text { [15\% MRI negativ] }\end{array}$ & $\begin{array}{l}0 \% \text { chr. } \\
\text { iEEG } \\
56 \% \text { akut } \\
\text { ECoG }\end{array}$ & $\begin{array}{l}\text { Anfallsfreiheit } 68 \% \text {, Med. gestoppt n/a } \\
\text { MEG: kontralaterale, okzipitale MEG Dipole }\end{array}$ \\
\hline $\begin{array}{l}\text { Davis [56] } \\
\text { (2012) } \\
\text { Seizure } \\
\text { USA }\end{array}$ & $\begin{array}{l}43 \text { Pt, gemischt } \\
\text { Alter: } \mathrm{n} / \mathrm{a}(20,9) \\
\text { Rekr: } 1983-2008 \\
\text { Nachkontr: } 1 \mathrm{~J}\end{array}$ & $\begin{array}{l}\text { O und benachbarte } \mathrm{P} / \mathrm{T} \\
28 \% \text { Tumor } \\
{[19 \% \mathrm{MRI} \text { negativ] }}\end{array}$ & $\begin{array}{l}76 \% \text { chr. } \\
\text { iEEG } \\
2 \% \text { akut } \\
\text { ECoG }\end{array}$ & $\begin{array}{l}\text { Anfallsfrei } 51 \% \text {, Med. gestoppt: } 0 \% \\
\text { Semiologie: keine Kopfversion ohne visuelle Aura, keine temporale Aura } \\
\text { EEG: fokales interiktales Oberflächen-EEG } \\
\text { Ätiologie: niedergradiger Tumor, multilobäre FCD }\end{array}$ \\
\hline $\begin{array}{l}\text { Dorfer [57] } \\
\text { (2013) } \\
\text { Neurosurgery } \\
\text { Focus } \\
\text { Österreich }\end{array}$ & $\begin{array}{l}10 \text { Pt, gemischt } \\
\text { Alter: } 4-22(8,7) \\
\text { Rekr: n/a } \\
\text { Nachkontr: } 2 \mathrm{~J}\end{array}$ & $\begin{array}{l}\mathrm{TPO}^{\mathrm{b}} \\
50 \% \mathrm{FCD}\end{array}$ & $\begin{array}{l}10 \% \text { chr. } \\
\text { iEEG } \\
0 \% \text { akut } \\
\text { ECoG }\end{array}$ & $\begin{array}{l}\text { Anfallsfreiheit } 90 \% \text {, Med. gestoppt: } 10 \% \\
\text { Statistische Auswertung prädiktiver Faktoren nicht angegeben }\end{array}$ \\
\hline $\begin{array}{l}\text { Liava [37] } \\
\text { (2014) } \\
\text { Epilept Disord } \\
\text { Italien }\end{array}$ & $\begin{array}{l}62 \text { Pt, pädiatrisch } \\
\text { Alter: } 1-16(7,9) \\
\text { Rekr: } 1996-2011 \\
\text { Nachkontr: } 6,9 \mathrm{~J}\end{array}$ & $\begin{array}{l}\text { P, O, OP, TO, TP } \\
50 \% \text { FCD, } 24 \% \text { Tumor, } \\
15 \% \text { Gliose }\end{array}$ & $\begin{array}{l}38 \% \text { chr. } \\
\text { iEEG } \\
\text { n/a akut } \\
\text { ECoG }\end{array}$ & $\begin{array}{l}\text { Anfallsfreiheit } 86 \% \text {, Med. gestoppt n/a } \\
\text { Anamnese: älter bei Epilepsiebeginn } \\
\text { MRl: begrenzte epileptogene Läsion }\end{array}$ \\
\hline $\begin{array}{l}\text { Craciun [58] } \\
\text { (2018) } \\
\text { Epilept Disord } \\
\text { Frankreich }\end{array}$ & $\begin{array}{l}16 \text { Pt, pädiatrisch } \\
\text { Alter: } 1-16(7,8) \\
\text { Rekr: } 2007-2014 \\
\text { Nachkontr: } 2,6 \mathrm{~J}\end{array}$ & $\begin{array}{l}\text { O, PO, TO, TPO } \\
56 \% \text { FCD, } 13 \% \text { Tumor, } \\
19 \% \text { Gliose } \\
{[0 \% \text { MRI negativ] }}\end{array}$ & $\begin{array}{l}100 \% \text { chr. } \\
\text { iEEG } \\
0 \% \text { akut } \\
\text { ECoG }\end{array}$ & $\begin{array}{l}\text { Anfallsfreiheit } 69 \% \text {, Med. gestoppt } \mathbf{n} / \mathbf{a} \\
\text { PET: fokaler Hypometabolismus }\end{array}$ \\
\hline $\begin{array}{l}\text { Ramantani } \\
\text { [19] (2017) } \\
\text { Epilepsia } \\
\text { Deutschland }\end{array}$ & $\begin{array}{l}50 \text { Pt, pädiatrisch } \\
\text { Alter: 1,5-18 (8) } \\
\text { Rekr: 1997-2014 } \\
\text { Nachkontr: } \\
\text { 1,5-18 J }\end{array}$ & $\begin{array}{l}\mathrm{P}, \mathrm{O}, \mathrm{PO}, \text { postT } \\
44 \% \mathrm{FCD}, 32 \% \text { Tumor, } \\
16 \% \text { postischämisch, } 8 \% \\
\text { tuberöse Sklerose } \\
\text { [1 } \% \text { MRI negativ] }\end{array}$ & $\begin{array}{l}26 \% \text { chr. } \\
\text { iEEG } \\
38 \% \text { akut } \\
\text { ECoG }\end{array}$ & $\begin{array}{l}\text { Anfallsfreiheit } 60 \% \text {, Med. gestoppt } 30 \% \\
\text { Anamnese: längere Epilepsiedauer }\end{array}$ \\
\hline
\end{tabular}


Sierra-Marcos $37 \mathrm{Pt}$, pädiatrisch [59] (2017) Alter: 0,3-23 (10)

Seizure

Spanien
$\mathrm{P}, \mathrm{O}, \mathrm{PO}$, postT $35 \%$ FCD, $11 \%$ Tumor, $18 \%$ postischämisch [3\% MRI negativ]
$43 \%$ chr. iEEG n/a akut ECoG
Anfallsfreiheit $62 \%$, Med. gestoppt $\mathrm{n} / \mathrm{a}$ Anamnese: niedrige Anfallsfrequenz, wenigere Antikonvulsiva. MRI: eng umschriebene Läsion, epileptogene Läsion nur im posterioren Kortex EEG: keine postoperativen epilepsietypischen Potenziale

FCD "focal cortical dysplasia" (fokale kortikale Dysplasie), J. Jahre, Med. Medikamente, O Okzipitallappen, $P$ Parietallappen, postT posteriorer Temporallappen, Pt. Patient, $n / a$ nicht angegeben, Nachkontr. Nachkontrolle, Rekr. Rekrutierungsintervall

${ }^{a}$ Ein Eingriff war multiple subpiale Transsektionen

${ }^{\mathrm{b}} 80 \%$ der Patienten erhielten TPO-Diskonnektionen

\section{Die Rolle multilobärer Resektionen}

Multilobäre Resektionen werden im Kindes- und Jugendalter viel häufiger [38-40] als bei Erwachsenen [41] durchgeführt und machen damit laut der internationalen Umfrage von 2004 $18 \%$ der pädiatrischen epilepsiechirurgischen Eingriffe aus [30]. Diese höhere Zahl multilobärer Resektionen reflektiert die höhere Prävalenz der oft ausgedehnten FCDs. Bei jüngeren Kindern ist die Wahrscheinlichkeit einer multilobären FCD im Vergleich zu älteren Patienten mit zumeist fokalen FCDs deutlich erhöht [42-44]. Von den 133 Kindern in der kürzlich publizierten Studie zur FCDassoziierten Epilepsiechirurgie [45] wurde bei $16 \%$ eine multilobäre Resektion durchgeführt.

Insgesamt und trotz der hohen Relevanz der multilobären Resektionen für die Epilepsiechirurgie in pädiatrischen Populationen sind diese viel weniger ausführlich untersucht und in der Literatur beschrieben als intralobäre oder hemisphärische Eingriffe. Die verfügbaren Daten stammen von Subgruppenanalysen als Teil großer pädiatrischer [38-40, 46 , 47] oder pädiatrisch/erwachsen gemischter [41] chirurgischer Fallserien. In diesen werden sehr variable Raten für eine Anfallsfreiheit von 18-60\% angegeben, welche teilweise auf schlechte postoperative Verläufe bei multilobären im Vergleich zu intralobären oder hemisphärischen Eingriffen hinweisen [38]. In diesen Studien waren linksseitige sowie frontotemporale und temporoparietale Resektionen, residuelle Läsionen im postoperativen MRI und epilepsietypi- sche Potenziale im postoperativen EEG [48, 49] mit einem erhöhten Risiko für ein Anfallsrezidiv assoziiert.

In einer kürzlich publizierten Kohorte, welche 69 Kinder und Jugendliche mit multilobären Resektionen beinhaltet, waren $48 \%$ der Patienten anfallsfrei bei der letzten Verlaufskontrolle nach 9 Jahren, und $33 \%$ wurden nicht mehr antikonvulsiv behandelt. Zehn Prozent der Patienten, alle mit einer dysplastischen Ätiologie, mussten erneut operiert werden: Der Großteil dieser Patienten erreichte jedoch dann Anfallsfreiheit. Anfallsfreiheit war stark mit einer kompletten Resektion sowohl der epileptogenen Zone als auch der Läsion assoziiert. Die Nähe der Läsion bzw. der epileptogenen Zone zu eloquentem Kortex stellte behandelnde Ärzte und Familien vor ein Dilemma. Gerade auch in Bezug auf die weiter oben illustrierten Schwierigkeiten bei der Evaluation posteriorer Epilepsien im Kindesalter stellen multilobäre Läsionen, die den posterioren Kortex involvieren, eine besondere Herausforderung bezüglich optimaler prächirurgischer $\mathrm{Ab}$ klärung und Patientenselektion dar.

\section{Der richtige Eingriff für das richtige Kind}

Bei älteren Kindern und Jugendlichen ist eine multimodale, umfassende nichtinvasive präoperative Abklärung in Kombination mit einer invasiven EEG-Ableitung ausschlaggebend sowohl für die akkurate Identifizierung der epileptogenen Zone als auch für ihre Abgrenzung gegenüber eloquenten kortikalen Arealen [50]. Bei jüngeren Kindern sind allerdings eine frühere Intervention und eine radikale- re und damit komplettere Resektion indiziert in Anbetracht der funktionellen Plastizität des frühen Kindesalters, die bei der Kompensation erwarteter funktioneller Defizite offensichtliche Vorteile bietet, wie von Neumayr et al. in dieser Ausgabe zusammengefasst. In vielen Studien - sowohl spezifisch zu posterioren Epilepsien (•Tab. 1) als auch zur pädiatrischen Epilepsiechirurgie allgemein [31] - ist eine kürzere Epilepsiedauer mit höheren Chancen auf eine postoperative Anfallsfreiheit assoziiert. Diese kann nur erreicht werden, wenn Kinder mit Epilepsie und strukturellen Läsionen - auch bei epilepsiechirurgisch komplexeren Fällen wie bei Epilepsien im posterioren Kortex - frühzeitig und umfangreich prächirurgisch abgeklärt werden.

\section{Korrespondenzadresse}

Dr. Richard E. Rosch, MRCPCH MD PhD Neuropädiatrie, Universitäts-Kinderspital Zürich

Steinwiesstr. 75, 8032 Zürich, Schweiz richard.rosch@kispi.uzh.ch

Funding. Open access funding provided by University of Zurich

\section{Einhaltung ethischer Richtlinien}

Interessenkonflikt. R. Rosch, A. Rüegger und G. Ramantani geben an, dass kein Interessenkonflikt besteht.

Für diesen Beitrag wurden von den Autoren keine Studien an Menschen oder Tieren durchgeführt. Für die aufgeführten Studien gelten die jeweils dort angegebenen ethischen Richtlinien.

Open Access. Dieser Artikel wird unter der Creative Commons Namensnennung 4.0 International Lizenz veröffentlicht, welche die Nutzung, Vervielfältigung, 
Bearbeitung, Verbreitung und Wiedergabe in jeglichem Medium und Format erlaubt, sofern Sie den/die ursprünglichen Autor(en) und die Quelle ordnungsgemäß nennen, einen Link zur Creative Commons Lizenz beifügen und angeben, ob Änderungen vorgenommen wurden.

Die in diesem Artikel enthaltenen Bilder und sonstiges Drittmaterial unterliegen ebenfalls der genannten Creative Commons Lizenz, sofern sich aus der Abbildungslegende nichts anderes ergibt. Sofern das betreffende Material nicht unter der genannten Creative Commons Lizenz steht und die betreffende Handlung nicht nach gesetzlichen Vorschriften erlaubt ist, ist für die oben aufgeführten Weiterverwendungen des Materials die Einwilligung des jeweiligen Rechteinhabers einzuholen.

Weitere Details zur Lizenz entnehmen Sie bitte der Lizenzinformation auf http://creativecommons.org/ licenses/by/4.0/deed.de.

\section{Literatur}

1. Aaberg KM, Gunnes N, Bakken IJ et al (2017) Incidence and prevalence of childhood epilepsy: a nationwide cohort study. Pediatrics 139:e20163908

2. Arts WFM, Geerts AT (2009) When to start drug treatment for childhood epilepsy: the clinicalepidemiological evidence. Eur J Paediatr Neurol 13:93-101

3. Berg AT, Mathern GW, Bronen RA et al (2009) Frequency, prognosis and surgical treatment of structural abnormalities seen with magnetic resonance imaging in childhood epilepsy. Brain 132:2785-2797

4. Kadish NE, Bast T, Reuner G et al (2019) Epilepsy surgery in the first 3 years of life: predictors of seizure freedom and cognitive development. Neurosurgery 84(6):E368-E377. https://doi.org/ $10.1093 /$ neuros/nyy376

5. Lamberink HJ, Boshuisen K, van Rijen PC, Gosselaar PH, Braun KPJ (2015) Dutch collaborative epilepsy surgery program (DCESP). Changing profiles of pediatric epilepsy surgery candidates over time: a nationwide single-center experience from 1990 to 2011. Epilepsia 56:717-725

6. Baud MO, Perneger T, Rácz A et al (2018) European trends in epilepsy surgery. Neurology 91:e96-e106

7. Cloppenborg T, May TW, Blümcke I et al (2019) Differences in pediatric and adult epilepsy surgery: a comparison at one center from 1990 to 2014. Epilepsia 60:233-245

8. Pestana Knight EM, Schiltz NK, Bakaki PM, Koroukian SM, Lhatoo SD, Kaiboriboon K (2015) Increasing utilization of pediatric epilepsy surgery in the United States between 1997 and 2009. Epilepsia 56:375-381

9. Dwivedi R, Ramanujam B, Chandra PS et al (2017) Surgery for drug-resistant epilepsy in children. NEngl J Med 377:1639-1647

10. Honda R, KaidoT, Sugai Ketal (2013) Long-term developmental outcome afterearly hemispherotomy for hemimegalencephaly in infants with epileptic encephalopathy. Epilepsy Behav 29:30-35

11. Loddenkemper T, Holland KD, Stanford LD, Kotagal P, Bingaman W, Wyllie E (2007) Developmental outcome after epilepsy surgery in infancy. Pediatrics 119:930-935

12. Skirrow C, Cross JH, Cormack F, Harkness W, Vargha-Khadem F, Baldeweg T (2011) Long-term intellectual outcome after temporal lobe surgery in childhood. Neurology 76:1330-1337

13. Skirrow C, Cross JH, Harrison Setal (2015) Temporal lobe surgery in childhood and neuroanatomical predictors of long-term declarative memory outcome. Brain 138:80-93

14. Kadish NE, Bast T, Reuner G et al (2019) Epilepsy surgery in the first 3 years of life: predictors of seizure freedom and cognitive development. Neurosurgery 84:E368-E377

15. Ramantani G, Kadish NE, Mayer H et al (2018) Frontal lobe epilepsy surgery in childhood and adolescence: predictors of long-term seizure freedom, overall cognitive and adaptive functioning Neurosurgery 83:93-103

16. Jonas R, Asarnow RF, LoPresti C et al (2005) Surgery for symptomatic infant-onset epileptic encephalopathy with and without infantile spasms. Neurology 64:746-750

17. Pulsifer MB, Brandt J, Salorio CF, Vining EPG, Carson BS, Freeman JM (2004) The cognitive outcome of hemispherectomy in 71 children. Epilepsia 45:243-254

18. Ramantani G, Kadish NE, Anastasopoulos C et al (2014) Epilepsy surgery for glioneuronal tumors in childhood: avoid loss of time. Neurosurgery 74:648-657 (discussion 657)

19. Ramantani G, Stathi A, Brandt A et al (2017) Posterior cortex epilepsy surgery in childhood and adolescence: predictors of long-term seizure outcome. Epilepsia 58:412-419

20. Simasathien T, Vadera S, Najm I, Gupta A, Bingaman W, Jehi L (2013) Improved outcomes with earlier surgery for intractable frontal lobe epilepsy. Ann Neurol 73:646-654

21. Rosenow F, Lüders H (2001) Presurgical evaluation of epilepsy. Brain 124:1683-1700

22. Maillard LG, Tassi L, Bartolomei Fet al (2017) Stereoelectroencephalography and surgical outcome in polymicrogyria-related epilepsy: a multi-centric study. Ann Neurol 82(5):781-794. https://doi.org/ 10.1002/ana.25081

23. Wyllie E, Lachhwani DK, Gupta A et al (2007) Successful surgery for epilepsy due to early brain lesions despite generalized EEG findings. Neurology 69:389-397

24. Ramantani G, Kadish NE, Strobl K et al (2013) Seizure and cognitive outcomes of epilepsy surgery in infancy and early childhood. Eur J Paediatr Neurol 17:498-506

25. Ramantani G (2019) Epilepsy surgery in early life: the earlier, the better. World Neurosurg 131:285-286

26. HamerHM, Wyllie E, Lüders HO, KotagalP, Acharya J (1999) Symptomatology of epileptic seizures in the first three years of life. Epilepsia 40:837-844

27. Eltze CM, Chong WK, Bhate S, Harding B, Neville BGR, Cross JH (2005) Taylor-type focal cortical dysplasia in infants: some MRI lesions almost disappear with maturation of myelination. Epilepsia 46:1988-1992

28. Ryvlin P, Cross JH, Rheims S (2014) Epilepsy surgery in children and adults. Lancet Neurol 13:1114-1126

29. Braun KPJ, Cross JH (2018) Pediatric epilepsy surgery: the earlier the better. Expert Rev Neurother 18:261-263

30. Harvey AS, Cross JH, Shinnar S, Mathern GW, Mathern BW, ILAE Pediatric Epilepsy Surgery Survey Taskforce (2008) Defining the spectrum of international practice in pediatric epilepsy surgery patients. Epilepsia 49:146-155

31. Englot DJ, Breshears JD, Sun PP, Chang EF, Auguste KI (2013) Seizure outcomes after resective surgery for extra-temporal lobe epilepsy in pediatric patients. J Neurosurg Pediatr 12:126-133

32. D'Argenzio L, Colonnelli MC, Harrison Set al (2012) Seizure outcome after extratemporal epilepsy surgery in childhood. Dev Med Child Neurol 54:995-1000

33. Liava A, Francione S, Tassi L et al (2012) Individually tailored extratemporal epilepsy surgery in children: anatomo-electro-clinical features and outcome predictors in a population of 53 cases. Epilepsy Behav 25:68-80

34. Blount JP (2017) Extratemporal resections in pediatricepilepsysurgery-anoverview. Epilepsia 58(1):19-27

35. Ibrahim GM, Fallah A, Albert GW et al (2012) Occipital lobe epilepsy in children: characterization, evaluation and surgical outcomes. Epilepsy Res 99:335-345

36. Yang P-F, Mei Z, Lin Q et al (2014) Disconnective surgery in posterior quadrantic epilepsy: a series of 12 paediatric patients. Epileptic Disord 16:296-304

37. Liava A, Mai R, Tassi Letal (2014) Paediatric epilepsy surgery in the posterior cortex: a study of 62 cases. Epileptic Disord 16:141-164

38. Paolicchi JM, Jayakar P, Dean P et al (2000) Predictors of outcome in pediatric epilepsy surgery. Neurology 54:642-647

39. Hemb M, Velasco TR, Parnes MS et al (2010) Improved outcomes in pediatric epilepsy surgery: the UCLA experience, 1986-2008. Neurology 74:1768-1775

40. Teutonico F, Mai R, Veggiotti P et al (2013) Epilepsy surgery in children: evaluation of seizure outcome and predictive elements. Epilepsia 54(7):70-76

41. Edelvik A, Rydenhag B, Olsson I et al (2013) Longterm outcomes of epilepsy surgery in Sweden: a national prospective and longitudinal study. Neurology 81:1244-1251

42. Cepeda C, André VM, Levine MS et al (2006) Epileptogenesis in pediatric cortical dysplasia: the dysmature cerebral developmental hypothesis. Epilepsy Behav 9:219-235

43. Lerner JT, Salamon N, Hauptman JS et al (2009) Assessment and surgical outcomes for mild type I and severe typell cortical dysplasia: a critical review and the UCLA experience. Epilepsia 50:1310-1335

44. Hauptman JS, Mathern GW (2012) Surgical treatment of epilepsy associated with cortical dysplasia: 2012 update. Epilepsia 53(4):98-104

45. Martinez-Lizana E, Fauser S, Brandt A et al (2018) Long-term seizure outcome in pediatric patients with focal cortical dysplasia undergoing tailored and standard surgical resections. Seizure 62:66-73

46. Cossu M, Lo Russo G, Francione $S$ et al (2008) Epilepsy surgery in children: results and predictors of outcome on seizures. Epilepsia 49:65-72

47. Jayakar P, Dunoyer C, Dean P et al (2008) Epilepsy surgery in patients with normal or nonfocal MRI scans: integrative strategies offer long-term seizure relief. Epilepsia 49:758-764

48. Sarkis RA, Jehi L, Najm IM, Kotagal P, Bingaman WE (2012) Seizure outcomes following multilobar epilepsy surgery. Epilepsia 53:44-50

49. Cho EB, Joo EY, Seo D-W, Hong S-C, Hong SB (2015) Prognostic role of functional neuroimaging after multilobar resection in patients with localizationrelated epilepsy. PLoS ONE 10:e136565

50. Kogias E, Schmeiser B, Doostkam S et al (2019) Multilobar resections for $3 t$ mri-negative epilepsy: worth the trouble? World Neurosurg 123:e338-e347

51. Boesebeck F, Schulz R, May T, Ebner A (2002) Lateralizing semiology predicts the seizure 
outcome after epilepsy surgery in the posterior cortex. Brain 125:2320-2331

52. Barba C, Doglietto F, De Luca L et al (2005) Retrospective analysis of variables favouring good surgical outcome in posterior epilepsies. J Neurol 252:465-472

53. Dalmagro CL, Bianchin MM, Velasco TR et al (2005) Clinical features of patients with posterior cortex epilepsies and predictors of surgical outcome. Epilepsia 46:1442-1449

54. Jehi LE, O'Dwyer R, Najm I, Alexopoulos A, Bingaman W (2009) A longitudinal study of surgical outcome and its determinants following posterior cortex epilepsy surgery. Epilepsia 50:2040-2052

55. Yu T, Wang Y, Zhang G, Cai L, Du W, Li Y (2009) Posterior cortex epilepsy: diagnostic considerations and surgical outcome. Seizure 18:288-292

56. Davis KL, Murro AM, Park YD, Lee GP, Cohen MJ, Smith JR (2012) Posterior quadrant epilepsy surgery: predictors of outcome. Seizure 21:722-728

57. Dorfer $C$, Czech T, Mühlebner-Fahrngruber A et al (2013) Disconnective surgery in posterior quadrantic epilepsy: experience in a consecutive series of 10 patients. Neurosurg Focus 34:E10

58. Craciun L, Taussig D, Ferrand-Sorbets S et al (2018) Investigation of paediatric occipital epilepsy using stereo-EEG reveals a better surgical outcome than in adults, especially when the supracalcarine area is affected. Epileptic Disord 20:346-363

59. Sierra-Marcos A, Castillo MCF, Álvarez-Linera J, Budke M, García-Fernández M, Pérez-Jiménez MA (2017) Functional surgery in pediatric drugresistant posterior cortex epilepsy: electro-clinical findings, cognitive and seizure outcome. Seizure 52:46-52

\section{Oğuz Çataltepe, George I Jallo (eds) Pediatric Epilepsy Surgery}

\section{Thieme 2020, 2. Auflage, 695 S., 796 Abb., (ISBN: 978-1-62623-816-9), Hardcover 269,99 EUR}

Die beiden Herausgeber des nach zehn Jahre neu aufgelegten, großformatigen Buches sind ausgewiesene Experten. Oğuz Çataltepe $\left({ }^{*} 1959\right)$ ist ein türkischstämmiger USamerikanischer pädiatrischer Neurochirurg und Associate Professor in der Neurochirurgie der Departments of Surgery and Pediatrics sowie Leiter der Pädiatrischen Neurochirurgie und Ko-Leiter der Epilepsiechirurgie an der University of Massachusetts Medical School in Worcester, Massachusetts (1). George Jallo $*^{*}$ 1966) ist ein in Isral geborener US-amerikanischer pädiatrischer Neurochirurg, der seine Facharztweiterbildung und Fellowship am New York University Medical Center in New York absolvierte und danach bis 2003 Assistant Professor of Neurosurgery and Pediatrics am Albert Einstein College of Medicine sowie Attending Neurosurgeon am Beth Israel Medical Center in New York war: Seitdem ist er am Johns Hopkins All Children's Institute for Brain Protection Sciences (IBPS) der Johns Hopkins University in Baltimore, Maryland, inzwischen als Leiter der gesamten Pädiatrischen Neurochirurgie sowie seit 2015 als Medizinischer Direktor des IBPS (2). Sie haben über 150 Mitarbeiterinnen und Mitarbeiter für das Buch gewinnen können, überwiegend aus Nordamerika und nur mit vereinzelter deutschsprachiger oder ursprünglich deutschsprachiger Beteiligung (Tobias Loddenkemper, Johannes Schramm, Margitta Seeck und Ingrid Tuxhorn).

Inhaltlich ist das Buch in fünf Hauptabschnitte gegliedert:

1. Einführung in die Pädiatrische Epileptologie (ca. 75 Druckseiten),

2. Präoperative Diagnostik inklusive Neuropsychologie, Elektrophysiologie (inkl. Stereo-EEG und MEG) und Bildgebung (inkl. funktioneller MRT, PET, iktalem SPECT sowie multimodaler Bildgebung und Koregistrierung) (ca. 100 Druckseiten);

3. Chirurgische Anatomie und Mapping-Techniken (ca. 80 Druckseiten),

4. Chirurgische Therapie inkl. Anästhesie, intrakranielle Elektrodenplatzierung, chirurgische Zugangswege, Hemisphärenchirurgie, andere diskonnektive Techniken, Neuromodulationsverfahren; Radiochirurgie und Ablationsverfahren (mit stereotaktischer Laserablation und MRTgestützter Thermokoagulation) (ca. 350 Druckseiten), und 5: Postoperativer Verlauf und Outcome (ca. 20 Druckseiten).

Im Kapitel zur postoperativen Anfallskontrolle wird leider an der Engel-Klassifikation (3) mit der Unterkategorie I einschließlich "nicht behindernder" Anfälle festgehalten. Es ist allerhöchste Zeit, diese entweder auf die Kategorie la (komplett anfallsfrei) zu beschränken oder durch die Wieser-Klassifikation (4) zu ersetzen, auch wenn diese die Daten in einem etwas weniger vorteilhaften Licht erscheinen lässt.

Die Ausstattung des Buches ist hervorragend, der Preis sicher für manche Assistenzärzte etwas prohibitiv. Das Buch sollte aber zumindest in keiner Präsenzbibliothek von Kliniken fehlen, die sich mit pädiatrischer Epilepsiechirurgie beschäftigen.

Günter Krämer, Zürich

1) https://physicians.umassmemorial.org/details/2366/oguz-cataltepe-neurological_surgeryspine-surgery-worcester; letzter Zugriff am 5. April 2020

2) https://www.hopkinsmedicine.org/profiles/results/directory/profile/0018539/george-jallo; letzter Zugriff am 5. April 2020

3) Engel J Jr (1987) Outcome with respect to epileptic seizures. In: Engel J Jr, ed. Surgical Treatment of the Epilepsies. New York, Raven Press: 553-572

4) Wieser HG, Blume WT, Fish D, et al; Commission on Neurosurgery of the International League Against Epilepsy (ILAE) (2001) Proposal for a new classification of outcome with respect to epileptic seizures following epilepsy surgery. ILAE Commission Report. Epilepsia 42: 282-286 